\title{
Efficiencies and System Temperature for a Beamforming Array
}

\author{
Karl F. Warnick and Brian D. Jeffs
}

\begin{abstract}
For an active beamforming array, standard definitions for efficiencies and system temperature are not available. We use noise considerations to generalize the single-antenna conventions for aperture efficiency, spillover efficiency, radiation efficiency, and system temperature to arrays. The treatment leads to a new noise matching efficiency that quantifies the effect of mutual coupling on amplifier noise. Numerical results for a phased array feed indicate that the noise increase caused by mutual coupling can be significant.
\end{abstract}

\section{INTRODUCTION}

The primary figure of merit for a receiving antenna system is the sensitivity, which is related to the output SNR by

$$
\frac{A_{e}}{T_{\mathrm{sys}}}=\frac{k_{b} B}{S^{\mathrm{sig}}} \mathrm{SNR}
$$

where $S^{\mathrm{sig}}\left(\mathrm{W} / \mathrm{m}^{2}\right)$ is the signal power density in one polarization and $k_{b}$ is Boltzman's constant. For passive antennas, the sensitivity is commonly subdivided into signal and noise contributions using aperture efficiency, equivalent system temperature, and other figures of merit, but for arrays, adequate definitions of these parameters are unavailable. The goal of this paper is to generalize the standard definitions for aperture, spillover, and radiation efficiencies and system temperature to an active beamforming array.

Partial results on efficiency and system temperature definitions for arrays are available in the literature. Solidbeam efficiency is well defined for receiving arrays, but although this quantity is closely related to aperture efficiency, the two parameters are not equivalent and the solid-beam efficiency does not provide a satisfactory generalization. For transmitting arrays, the element efficiency [1] is useful for quantifying mismatch loss. The element efficiency can be used to parameterize mutual coupling effects for active receiving arrays, but it does not account for the effect of mutual coupling on front end amplifier noise. Craeye has developed an integral formulation for the contribution of thermal noise to the system temperature of a receiving array [2].

The authors are with the Department of Electrical and Computer Engineering, 459 Clyde Building, Brigham Young University, Provo, UT 84602.
In this paper, we show that noise considerations can be used to generalize the complete family of single antenna efficiencies to the array case. In [3], a definition for the aperture efficiency of a receiving array was developed. Here, we extend the analysis to include receiver noise and antenna ohmic losses. The treatment leads to a new noise matching efficiency that quantifies the effect of array mutual coupling on amplifier noise. Numerical results are given for a phased array feed.

Narrowband processing with center frequency $\omega$ and noise equivalent bandwidth $B$ is assumed. Field vectors are denoted with an overbar $(\bar{E})$ and vectors of array output voltages in bold (v). All voltages and fields are phasors relative to $e^{j \omega t}$.

\section{Array Signal And Noise Model}

An array can be characterized by an impedance matrix $\mathbf{Z}_{A}$ and the open circuit voltages $v_{o c, m}\left(\hat{p}, E_{0}, \Omega\right)$ induced at the element ports by a plane wave with polarization $\hat{p}$, electric field intensity $E_{0}$, and spherical angle of arrival $\Omega$. We will arrange these voltages into a column vector $\mathbf{v}_{\mathrm{Oc}}$. By reciprocity, it can be shown that $[4,5]$

$$
v_{o c, m}\left(\hat{p}, E_{0}, \Omega\right)=\frac{4 \pi j r e^{j k r}}{\omega \mu I_{0}} E_{0} \hat{p} \cdot \bar{E}_{m}(\bar{r})
$$

where the position vector $\bar{r}$ has polar coordinates $(r, \Omega)$ and $\bar{E}_{m}(\bar{r})$ is the far electric field radiated with input current $I_{0}$ into the $m$ th array element and all other elements open circuited (i.e., the embedded open circuit loaded radiated field pattern). For an array feed, the patterns are in the presence of a reflector, although in some computations the bare array patterns can be substituted with little loss in accuracy. Using (2), the array response can be expressed either in terms of the received voltage patterns $v_{o c, m}\left(\hat{p}, E_{0}, \Omega\right)$ or the radiated field patterns $\bar{E}_{m}(\bar{r})$.

Each array element is connected to a low noise amplifier and receiver chain. Voltages at the receiver outputs are related to the open circuit voltages by a linear transformation

$$
\mathbf{v}=\mathbf{Q} \mathbf{v}_{\mathrm{oc}}
$$

If the array is loaded by a network with impedance matrix $\mathbf{Z}_{R}$, and the receiver chains are identical, uncoupled, and have voltage gain $g$, then $\mathbf{Q}=g \mathbf{Z}_{R}\left(\mathbf{Z}_{R}+\mathbf{Z}_{A}\right)^{-1}$. 
The receiver output signals are combined in analog or digital processing using a vector $\mathbf{w}$ of complex weights to produce one scalar output signal per beam. The beamformer output is

$$
v=\mathbf{w}^{H} \mathbf{v}
$$

The beam output consists of a signal of interest as well as noise due to external thermal radiation, ohmic losses, and receivers. It is convenient to represent these contributions in terms of correlation matrices. If the environment is statistically wide sense stationary, the receiver output correlation matrix is

$$
\mathbf{R}_{\mathbf{v}}=\mathrm{E}\left[\mathbf{v v}^{H}\right]=\lim _{N \rightarrow \infty} \frac{1}{N} \sum_{n=1}^{N} \mathbf{v}[n] \mathbf{v}^{H}[n]
$$

where $n$ represents a sample index. The time average beam output power is proportional to $\mathbf{w}^{H} \mathbf{R}_{\mathbf{v}} \mathbf{w}$, and the beam signal to noise ratio (SNR) is

$$
\mathrm{SNR}=\frac{\mathbf{w}^{H} \mathbf{R}_{\text {sig }} \mathbf{w}}{\mathbf{w}^{H}\left(\mathbf{R}_{\text {ext }}+\mathbf{R}_{\text {loss }}+\mathbf{R}_{\text {rec }}\right) \mathbf{w}}
$$

where the correlation matrices are defined below.

The beam output power due to a point source signal of interest with angle of arrival $\Omega_{\text {sig }}$ is $P_{\text {sig }}=\mathbf{w}^{H} \mathbf{R}_{\text {sig }} \mathbf{w}$, where the signal correlation matrix is

$$
\mathbf{R}_{\text {sig }}=\mathbf{Q} \mathbf{v}_{\text {sig }, o c} \mathbf{v}_{\text {sig }, o c}^{H} \mathbf{Q}^{H}
$$

and $\mathbf{v}_{\text {sig,oc }}=\mathbf{v}_{\text {oc }}\left(\hat{p}_{\text {sig }}, E_{0, \text { sig }}, \Omega_{\text {sig }}\right)$. The receiver noise power at the beamformer output is $P_{\text {rec }}=\mathbf{w}^{H} \mathbf{R}_{\text {rec }} \mathbf{w}$, where $\mathbf{R}_{\text {rec }}$ is the correlation matrix of the receiver noise at the receiver outputs. Due to mutual coupling, thermal noise from one front end amplifier couples through the array into the other receiver channel inputs, so the receiver noise is correlated and $\mathbf{R}_{\text {rec }}$ is nondiagonal. $\mathbf{R}_{\text {rec }}$ can be modeled using network theory and amplifier noise parameters [6].

From Eq. (13) of [3], the correlation matrix of the open circuit voltages due to external thermal radiation from the warm scene around the array has elements given by

$$
R_{\mathrm{ext}, \mathrm{mn}}^{\mathrm{oc}}=\frac{8 k_{b} B}{\eta_{0}\left|I_{0}\right|^{2}} \int T(\Omega) \bar{E}_{m}(\bar{r}) \cdot \bar{E}_{n}^{*}(\bar{r}) r^{2} d \Omega
$$

where $T(\Omega)$ is the brightness temperature distribution. For an array feed, external thermal noise arrives from a solid angle $\Omega_{\mathrm{sp}}$, which is the complement of the solid angle subtended by the reflector relative to the feed. Typically, the background temperature distribution is nonuniform, but in order for the spillover efficiency definition given later to coincide with the IEEE convention, $T(\Omega)$ must be taken to be a constant $T_{\mathrm{sp}}$ over $\Omega_{\mathrm{sp}}$. Using (8) for this temperature distribution leads to

$$
\mathbf{R}_{\mathrm{ext}}=\mathbf{R}_{\mathrm{sp}}=\frac{1}{\left|I_{0}\right|^{2}} 16 k_{b} T_{\mathrm{sp}} B \mathbf{Q} \mathbf{A}_{\mathrm{sp}} \mathbf{Q}^{H}
$$

where

$$
A_{\mathrm{sp}, m n}=\frac{1}{2 \eta_{0}} \int_{\Omega_{\mathrm{sp}}} \bar{E}_{m}(\bar{r}) \cdot \bar{E}_{n}^{*}(\bar{r}) r^{2} d \Omega
$$

and in this formula the array feed element patterns $\bar{E}_{n}$ are in the absence of the reflector, which implies that sky noise in the reflector pattern main lobe is neglected. This simple spillover noise model is necessary for the purposes of this paper, but (8) can be used to include nonuniform sky and ground temperature distributions if needed.

To determine the noise due to antenna element ohmic losses, it is convenient to consider the case of the array in thermal equilibrium with an isotropic noise environment at temperature $T_{\text {iso. }}$. In this situation, the total thermal noise correlation matrix is [7]

$$
\mathbf{R}_{\mathrm{t}}=8 k_{b} T_{\text {iso }} B \mathbf{Q} \operatorname{Re}\left[\mathbf{Z}_{A}\right] \mathbf{Q}^{H}
$$

This expression is larger by a factor of two than in [7] due to a different phasor convention. From (8) with $T(\Omega)=T_{\text {iso }}$, the contribution from the external isotropic thermal noise alone is

$$
\mathbf{R}_{\text {iso }}=\frac{1}{\left|I_{0}\right|^{2}} 16 k_{b} T_{\text {iso }} B \mathbf{Q A A} \mathbf{Q}^{H}
$$

where $\mathbf{A}$ is the pattern overlap integral matrix with elements given by

$$
A_{m n}=\frac{1}{2 \eta_{0}} \int \bar{E}_{m}(\bar{r}) \cdot \bar{E}_{n}^{*}(\bar{r}) r^{2} d \Omega
$$

The difference between (11) and (12) gives the noise contribution due to ohmic losses in the antenna elements,

$$
\mathbf{R}_{\text {loss }}=8 k_{b} T_{\mathrm{a}} B \mathbf{Q R} \mathbf{R}_{A, \text { ohmic }} \mathbf{Q}^{H}
$$

where $\mathbf{R}_{A \text {,ohmic }}=\operatorname{Re}\left[\mathbf{Z}_{A}\right]-2 \mathbf{A} /\left|I_{0}\right|^{2}$ and $T_{\mathrm{a}}$ is the physical temperature of the array elements. By combining (8) and (14), the total thermal noise correlation matrix can be obtained for any external temperature distribution $T(\Omega)$ and physical array temperature $T_{\mathrm{a}}$. Finally, we observe that while $\mathbf{R}_{\text {iso }}$ here is only a means to obtaining (14), it will be seen later to have a special importance in the receive array efficiency definitions.

\section{EFFICIENCY DEFINITIONS}

Formulas for the aperture and spillover efficiencies of an array feed were developed in [3]. We will briefly recap the spillover efficiency treatment and extend the aperture efficiency definition of [3] to the case of a lossy array. Radiation efficiency and a new noise matching efficiency will then be defined for the array.

For spillover and radiation efficiencies, the standard transmit-based definitions can be transfered to the receive case using reciprocity. Combining (3) and (4) shows that the beam output can be expressed in terms of open circuit voltages as $v=\hat{\mathbf{w}}^{H} \mathbf{v}_{\mathrm{oc}}$, where $\hat{\mathbf{w}}=\mathbf{Q}^{H} \mathbf{w}$. 
From (2), it follows that the radiation pattern of the array excited as a transmitter is identical to the receiving pattern if the element input currents are given by the vector $\mathbf{i}=\hat{\mathbf{w}}^{*}$. The total radiated power is

$$
P_{\text {rad }}=\frac{1}{\left|I_{0}\right|^{2}} \mathbf{i}^{H} \mathbf{A} \mathbf{i}=\frac{1}{\left|I_{0}\right|^{2}} \mathbf{w}^{H} \mathbf{Q} \mathbf{A} \mathbf{Q}^{H} \mathbf{w}
$$

For a transmitting array feed, with (10) and (15) the spillover efficiency is

$$
\eta_{\mathrm{sp}}=1-\frac{P_{\mathrm{sp}}}{P_{\mathrm{rad}}}=1-\frac{\mathbf{w}^{H} \mathbf{Q} \mathbf{A}_{\mathrm{sp}} \mathbf{Q}^{H} \mathbf{w}}{\mathbf{w}^{H} \mathbf{Q} \mathbf{A} \mathbf{Q}^{H} \mathbf{w}}
$$

For the receive case, we can use (9) and (12) to obtain

$$
\eta_{\text {sp }}=1-\frac{T_{\text {iso }}}{T_{\text {sp }}} \frac{\mathbf{w}^{H} \mathbf{R}_{\text {sp }} \mathbf{w}}{\mathbf{w}^{H} \mathbf{R}_{\text {iso }} \mathbf{w}}
$$

Defining aperture efficiency for a beamforming array is problematic, because it is not obvious how to define available power for an active multi-antenna system. A workable definition for available power can be obtained by observing that for a passive antenna in thermal equilibrium with an isotropic thermal noise field with brightness temperature $T_{\text {iso }}$, the available noise power in the bandwidth $B$ at the antenna terminals is $k_{b} T_{\text {iso }} B$. Scaling the beamformer output to have the same property, so that

$$
P_{\mathrm{a}}=\left[\frac{k_{b} T_{\text {iso }} B}{\mathbf{w}^{H} \mathbf{R}_{\text {iso }} \mathbf{w}}\right] \mathbf{w}^{H} \mathbf{R}_{\mathbf{v}} \mathbf{w}
$$

allows the beamformer output to be viewed as available power at the terminals of an equivalent passive antenna. The aperture efficiency is then

$$
\eta_{\text {ap }}=\frac{P_{\text {sig,a }}}{P_{\text {inc }}}=\frac{k_{b} T_{\text {iso }} B}{A_{\text {ap }} S^{\text {sig }}} \frac{\mathbf{w}^{H} \mathbf{R}_{\text {sig }} \mathbf{w}}{\mathbf{w}^{H} \mathbf{R}_{\text {iso }} \mathbf{w}}
$$

where $A_{\text {ap }}$ is the physical aperture area.

By reciprocity, it can be shown that (19) is consistent with the standard conventions of antenna theory. The partial directivity with respect to the polarization $\hat{p}$ of the equivalent transmit array defined above is

$$
D_{p}(\Omega)=\frac{4 \pi r^{2} \frac{1}{2 \eta}\left|\sum_{m} i_{m} \hat{p} \cdot \bar{E}_{m}(\bar{r})\right|^{2}}{\mathbf{i}^{H} \mathbf{A i}}
$$

Using (2), (12), and (19) with (20), it can be shown that

$$
\eta_{\mathrm{ap}} A_{\mathrm{ap}}=\frac{\lambda^{2}}{4 \pi} D_{p}
$$

which mirrors the classical result for single antennas.

The power accepted by a reciprocal transmitting array is $P_{\text {in }}=\frac{1}{2} \mathbf{i}^{H} \operatorname{Re}\left[\mathbf{Z}_{A}\right] \mathbf{i}$, with which the radiation efficiency can be written as

$$
\eta_{\mathrm{rad}}=\frac{P_{\mathrm{rad}}}{P_{\mathrm{in}}}=\frac{\frac{1}{\left|I_{0}\right|^{2}} \mathbf{w}^{H} \mathbf{Q} \mathbf{A} \mathbf{Q}^{H} \mathbf{w}}{\frac{1}{2} \mathbf{w}^{H} \mathbf{Q} \operatorname{Re}\left[\mathbf{Z}_{A}\right] \mathbf{Q}^{H} \mathbf{w}}
$$

Inserting (11) and (12) leads to an equivalent receive definition of radiation efficiency,

$$
\eta_{\text {rad }}=\frac{\mathbf{w}^{H} \mathbf{R}_{\text {iso }} \mathbf{w}}{\mathbf{w}^{H}\left(\mathbf{R}_{\text {iso }}+\mathbf{R}_{\text {loss }}\right) \mathbf{w}}
$$

which indicates that radiation efficiency for a receive array can be characterized by the increase in noise caused by antenna element ohmic losses, relative to the noise received from an isotropic external thermal environment.

These efficiencies can be used to decompose the receiver sensitivity into its various signal and noise contributions. Combining (6), (17), (19), and (22) with (1) leads to

$$
\frac{A_{e}}{T_{\mathrm{sys}}}=\frac{\eta_{\mathrm{rad}} \eta_{\mathrm{ap}} A_{\mathrm{ap}}}{\eta_{\mathrm{rad}}\left(1-\eta_{\mathrm{sp}}\right) T_{\mathrm{sp}}+\left(1-\eta_{\mathrm{rad}}\right) T_{\mathrm{a}}+T_{\mathrm{rec}}}
$$

where

$$
T_{\text {rec }}=T_{\text {iso }} \frac{\mathbf{w}^{H} \mathbf{R}_{\mathrm{rec}} \mathbf{w}}{\mathbf{w}^{H} \mathbf{R}_{\mathrm{t}} \mathbf{w}}
$$

is the beam equivalent receiver noise temperature.

\section{A. Noise Matching Efficiency}

Since all other terms in (23) include efficiency factors, it is natural to define an efficiency for the receiver noise as well. If the minimum equivalent noise temperature for one front end amplifier and receiver chain under optimal noise matching conditions is $T_{\min }$, a noise matching efficiency can be defined as

$$
\eta_{\mathrm{n}}=\frac{T_{\min }}{T_{\text {iso }}} \frac{\mathbf{w}^{H} \mathbf{R}_{\mathrm{t}} \mathbf{w}}{\mathbf{w}^{H} \mathbf{R}_{\mathrm{rec}} \mathbf{w}}
$$

This is a new figure of merit for a receiving array, which quantifies the increase in receiver noise caused by mutual coupling and mismatches between the antenna elements and front end amplifiers.

For an uncoupled array with each front end amplifier optimally noise matched to an antenna element, the noise matching efficiency is unity for all possible sets of beamforming weights (see [6]). If mutual coupling between array elements is significant, then for a given set of beamformer weights the amplifiers see effective, beam-dependent active impedances at the input ports [8]. If the amplifiers are noise matched to the active impedances, then $\eta_{\mathrm{n}}=1$ for that beam. For a different beam steering direction, the active impedances change, and the noise matching efficiency is less than unity.

Using (25), the beam sensitivity can be expressed in terms of dimensionless efficiencies as

$$
\frac{A_{e}}{T_{\mathrm{sys}}}=\frac{\eta_{\mathrm{rad}} \eta_{\mathrm{ap}} A_{\mathrm{ap}}}{\eta_{\mathrm{rad}}\left(1-\eta_{\mathrm{sp}}\right) T_{\mathrm{sp}}+\left(1-\eta_{\mathrm{rad}}\right) T_{\mathrm{a}}+T_{\mathrm{min}} / \eta_{\mathrm{n}}}
$$


The denominator, $T_{\mathrm{sys}}$, is now a combination of the ground temperature, physical array temperature, and minimum equivalent receiver noise temperature weighted by efficiency factors. For an aperture array (without reflector), (26) holds with $\left(1-\eta_{\mathrm{sp}}\right) T_{\mathrm{sp}}$ replaced by an external noise temperature $T_{\text {ext }}$ obtained from (8).

\section{NUMERICAL RESUltS}

To illustrate the proposed definitions for a specific example, we consider a phased array feed consisting of 19 thickened half-wave dipoles in a hexagonal array with $0.6 \lambda$ spacing at $1600 \mathrm{MHz}$. The array is backed by a circular ground plane at distance $\lambda / 4$ and is located at the focal plane of a paraboloidal reflector with $20 \mathrm{~m}$ diameter and $f / D=0.43$. The array elements are loaded by amplifiers with $T_{\min }=10 \mathrm{~K}$, noise resistance $R_{\mathrm{n}}=5 \Omega$, and optimal source impedance $Z_{\mathrm{opt}}$ equal to the antenna element embedded self impedances $(49-j 24 \Omega$ for the center element). The array was modeled as lossless, so $\eta_{\mathrm{rad}}=1$. Beamformer weights $\mathbf{w}$ were chosen to maximize the sensitivity (1) for each beam steering direction.

Figure 1 shows simulated sensitivity and efficiencies for this system. The aperture efficiency for the beam steered to boresight is $68 \%$ (without blockage, feed support scattering, or array calibration errors). At boresight, the beam equivalent receiver noise temperature is $16 \mathrm{~K}$ and the noise matching efficiency is $62 \%$. The noise matching efficiency is not a strong function of steering angle, because the beamformer weights move in a more or less fixed pattern across the array as the beam is steered, and the mutual impedances do not vary strongly in the interior of the array. These results are optimistic in that half-wave dipoles may be less strongly coupled than other types of array elements.

\section{CONClusion}

Using noise considerations, we have developed definitions for the efficiencies and system temperature of a formed beam for an active receiving array. We have expressed these definitions in terms of noise correlation matrices, but using fundamental relationships between the isotropic noise correlation matrix $\mathbf{R}_{\text {iso }}$, the element pattern overlap integral matrix $\mathbf{A}$, and the mutual resistance matrix $\operatorname{Re}\left[\mathbf{Z}_{A}\right]$, the definitions can be expressed equivalently in terms of impedance matrices, Sparameter matrices, or pattern overlap integrals (in fact, for a lossless, reciprocal array, $\mathbf{R}_{\text {iso }}, \mathbf{A}$, and $\operatorname{Re}\left[\mathbf{Z}_{A}\right]$ are all proportional).

The treatment motivated a new figure of merit for receiving arrays, the noise matching efficiency, which measures the increase in receiver noise caused by mismatch and mutual coupling. Numerical results show that
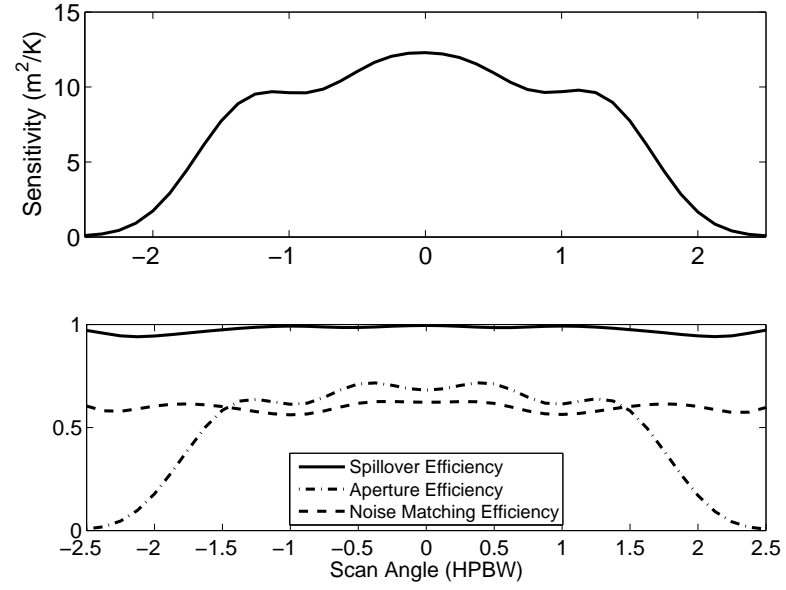

Fig. 1. Sensitivity, aperture efficiency, spillover efficiency, and noise matching efficiency for a 19 element phased array feed as a function of beam steering angle in half power beamwidths (HPBW) away from boresight.

for low noise applications such as phased array feeds for radio telescopes, the increase in receiver noise due to mutual coupling can cause an appreciable degradation in system performance.

\section{ACKNOWLEDGEMENTS}

We thank David Jones for providing numerical simulations for the array example and Marianna Ivashina for helpful discussions on efficiency definitions. This work was supported by the National Science Foundation grant number AST-9987339.

\section{REFERENCES}

[1] W. Kahn, "Element efficiency: a unifying concept for array antennas," IEEE Antennas Propag. Mag., vol. 49, pp. 48-56, Aug. 2007.

[2] C. Craeye, "Including spatial correlation of thermal noise in the noise model of high-sensivitity arrays," IEEE Trans. Ant. Propag., vol. 53, pp. 3845-3256, Nov. 2005.

[3] K. F. Warnick and B. D. Jeffs, "Gain and aperture efficiency for a reflector antenna with an array feed," IEEE Antennas and Wireless Propagation Letters, vol. 5, no. 1, pp. 499-502, 2006.

[4] A. T. De Hoop, "The N-port receiving antenna and its equivalent electrical network," Phillips Research Reports, vol. 30, pp. 302$315,1975$.

[5] P. S. Kildal, "Equivalent circuits of receive antennas in signal processing arrays," Microwave and Optical Technology Letters, vol. 21, no. 4, pp. 244-246, 1999.

[6] K. F. Warnick and M. A. Jensen, "Optimal noise matching for mutually-coupled arrays," IEEE Transactions on Antennas and Propagation, vol. 55, pp. 1726-1731, June 2007.

[7] R. Q. Twiss, "Nyquist's and Thevenin's theorems generalized for nonreciprocal linear networks," J. Applied Phys., vol. 26, pp. 599602, May 1955.

[8] E.E.M. Woestenburg, "Noise matching in dense phased arrays," Tech. Rep. RP-083, ASTRON, Dwingeloo, The Netherlands, Aug. 2005 . 